\title{
CLINICAL PRACTICE Review
}

\section{Update in New Medications for Primary Care}

\author{
Gerald W. Smetana, MD and Jane S. Sillman, $M D^{2}$
}

'Division of General Medicine and Primary Care, Beth Israel Deaconess Medical Center, Harvard Medical School, Boston, MA, USA; ${ }^{2}$ Division of General Internal Medicine and Primary Care, Brigham and Women's Hospital, Harvard Medical School, Boston, MA, USA.

J Gen Intern Med 25(3):261-9

DOI: $10.1007 / \mathrm{s} 11606-009-1209-0$

(c) Society of General Internal Medicine 2009

$\mathrm{E}$ ach year, many new drugs find their way into medical practice. Clinicians must determine which are suitable for primary care practice based on comparative efficacy, safety, and cost. Only a fraction of newly approved drugs each year are novel and relevant for primary care. Most new drugs each year are "me too" drugs within existing categories that have marginally differing properties when compared to currently available drugs. To illustrate this point we searched the FDA database for original new drug approvals, including new molecular entities and new biologics, for $2008^{1}$. We identified 51 new drug approvals. Twenty-six were significant new dosage forms, 10 were significant new biologicals, 10 were used primarily by specialists, 5 were new diagnostic agents (such as radiologic contrast media), and the remaining 6 were relevant for primary care. All six were "me too" drugs. Not a single new drug in 2008 was novel and relevant.

Therefore, we elected a different approach this year and identified drugs in the pipeline that are novel, have the potential to be relevant for primary care, and have generated substantial interest in the medical community. To develop a list of candidate drugs, we queried clinical pharmacists that consult for our institutional pharmacy and therapeutics committees, performed an internet search of lay media reports, and reviewed the following journals from January 1, 2008 through April 1, 2009: New England Journal of Medicine, Annals of Internal Medicine, ACP Journal Club, JAMA, Journal Watch, and the Lancet. We independently rated candidate drugs and developed our final list by consensus. In this paper, we will discuss anacetrapib to raise serum HDL levels, rivaroxaban for prevention of venous thromboembolism after surgery, and telcagepant to treat migraine headache.

For the second section of this paper, we present common clinical topics in primary care where the literature has provided new summary guidance on pharmacologic management and effective use of existing drugs. For this section, we used the same search strategy as that for novel drugs. This paper derives from a presentation at the $32^{\text {nd }}$ annual meeting of the Society of General Internal Medicine in May 2009.

Received August 24, 2009

Revised November 5, 2009

Accepted November 9, 2009

Published online December 12, 2009

\section{NEW DRUGS FOR PRIMARY CARE}

\section{Anacetrapib}

The introduction of statins to lower LDL and reduce cardiovascular risk has revolutionized the approach to primary and secondary prevention of cardiovascular disease. However, the impact of commonly used medications on serum HDL levels is modest; average increases range from $5-10 \%$ for statins to $15-$ $35 \%$ for niacin and fibric acid derivatives. Furthermore, while low serum HDL levels correlate with increased cardiovascular risk, few trials have addressed the impact of raising HDL levels on cardiovascular outcomes ${ }^{2}$.

Cholesterol ester transfer protein (CETP) is involved with reverse cholesterol transport of HDL from cholesterol laden macrophages (foam cells) to the liver and to other proteins. Mutations leading to lower CETP levels lead to markedly increased serum HDL levels and lower risk of cardiovascular events ${ }^{3,4}$. This observation encouraged research into pharmacologic inhibitors of CETP. The first such inhibitor to be extensively studied was torcetrapib. Early studies of torcetrapib demonstrated increases in serum HDL of 60-100\%. These observations prompted a large scale trial $(n=15,067)$ that evaluated clinical outcomes, the ILLUMINATE trial $^{5}$. In this trial, patients with untreated LDL levels of $\geq 100 \mathrm{mg} / \mathrm{dl}$ who had existing cardiovascular disease or type II diabetes were randomly assigned to atorvastatin (dose adjusted to achieve $\mathrm{LDL}<100 \mathrm{mg} / \mathrm{dl}$ ) or atorvastatin plus torcetrapib. While torcetrapib increased serum HDL levels by $72.1 \%$, there was an increase in cardiovascular events (HR 1.25, CI 1.09-1.44) and all cause mortality (HR 1.58, CI 1.14-2.19).

This unexpected finding raised questions as to whether a low HDL level is an actual cause of increased cardiovascular risk, or if it is an epiphenomenon. Alternatively, the particular drug, torcetrapib, has an aldosterone like effect, a so called "off-target effect," that may have been the cause for the observed increased mortality. This was suggested by the finding of increased blood pressure and decreased serum potassium levels in the patients receiving torcetrapib in the ILLUMINATE trial. This potential mechanism less easily explains the finding of a doubling in noncardiac deaths among patients receiving torcetrapib; therefore clinicians must be cautious attributing the increased all cause mortality solely to an aldosterone like effect.

Anacetrapib, a new CETP inhibitor that has no off-target effects was developed to test this hypothesis. Two published trials of anacetrapib have examined proxy endpoints. In a small phase I study, investigators examined the impact of anacetrapib on patients with dyslipidemia and on healthy volunteers ${ }^{6}$. In the first substudy, 50 patients with LDL levels from $100-190 \mathrm{mg} / \mathrm{dl}$ (100-160 mg/dl if 10-year coronary risk 10-20\%) were randomly assigned to placebo or one of 4 doses 
of anacetrapib (10, 40, 150 or $300 \mathrm{mg}$ daily) for 28 days. HDL levels rose in a dose dependent fashion from $41 \%$ to $129 \%$ for the highest dose (300 mg). Corresponding LDL reductions ranged from $5 \%$ to $38 \%$. In the same report, in a cohort of 22 healthy volunteers without hypertension, blood pressure measurements were nearly identical after 10 days of anacetrapib (150 mg) or placebo.

In a subsequent larger trial, eligible patients were adults aged 18-75 years with dyslipidemia defined by baseline coronary risk (100-190 mg/dl if average risk, $100-160 \mathrm{mg} / \mathrm{dl}$ if moderate risk, $100-130 \mathrm{mg} / \mathrm{dl}$ if diabetes) ${ }^{7}$. Importantly, patients with pre-existing coronary artery disease, symptomatic carotid artery disease, diabetes with an Alc $>8.5$, or hypertension were excluded. Bloomfield and colleagues randomly assigned 589 patients to one of 10 arms that included placebo, 4 doses of anacetrapib, atorvastatin $20 \mathrm{mg}$, or atorvastatin $20 \mathrm{mg}$ plus one of 4 doses of anacetrapib for 8 weeks. Among patients receiving anacetrapib, there were striking HDL elevations ranging from $44 \%$ to $133 \%$. The addition of atorvastatin did not significantly modify this effect. Anacetrapib also reduced LDL in a dose dependent fashion ranging from $16 \%$ to $39 \%$. The addition of atorvastatin to anacetrapib produced a synergistic effect on LDL reduction. There were no significant differences in median systolic or diastolic blood pressures between any of the groups. Serum potassium levels did not differ between placebo and each dose of anacetrapib. Anacetrapib was generally well tolerated with adverse effects that were similar to placebo.

Anacetrapib is the second CETP inhibitor to be studied in large clinical trials. It increases serum HDL to a greater extent than torcetrapib and by a factor of nearly four when compared to the currently available lipid lowering drugs. Anacetrapib appears to have succeeded in separating the CETP inhibition effect from the off-target aldosterone like effect. To the extent that the increased morbidity and mortality of torcetrapib was due solely to an aldosterone like effect, this suggests that anacetrapib has the potential to reduce cardiovascular morbidity without the unwanted off-target effects. However, whether pharmacologic manipulation of HDL levels leads to a reduction in morbidity and mortality is an unproven hypothesis. In addition, the available data do not allow one to definitively conclude that all adverse effects of torcetrapib were due to off target effects. Therefore, clinicians must be extremely cautious in expressing optimism or hope that anacetrapib will prove to be an important addition to our available therapies for patients with dyslipidemia. Large scale trials that measure clinical endpoints including cardiovascular morbidity and mortality, and all cause mortality will be necessary before concluding that CETP inhibition is an effective and safe strategy. If such studies show benefit and safety, this would be a welcome addition to our armamentarium. As of September 2009, the drug has not yet been submitted to the FDA.

\section{Rivaroxaban}

The risk of venous thromboembolism (VTE) after major orthopedic surgery is substantial in patients who are not receiving prophylaxis. For example, VTE rates after knee or hip replacement without prophylaxis range from $40 \%$ to $80 \%{ }^{8}$. As a result, thromboprophylaxis is routine after high risk surgery and most hospitals have developed institutional polices regarding their proper use. However, existing strategies all have potential disadvantages. Unfractionated heparin requires a continuous intravenous infusion, low molecular weight heparin is expensive and requires injections, and warfarin requires frequent monitoring and has a narrow therapeutic range. An ideal anticoagulant would be given orally, have a predictable anticoagulant response and therefore require no monitoring, have a rapid onset of action, have an antidote for safe and rapid reversal, and have no major side effects. Investigators have sought new targets for anticoagulation that would have many of these attributes. In addition to factor Xa inhibitors, long acting antithrombin activators and direct thrombin inhibitors are among new agents under study.

Rivaroxaban is a novel agent that works as a direct factor Xa inhibitor. Factor Xa works near the end of the coagulation cascade and catalyzes the conversion of factor II (prothrombin) to factor IIa (thrombin). Figure 1 illustrates the end of the coagulation cascade and the site of action of rivaroxaban. Rivaroxaban has many of the desired anticoagulant attributes. It has a rapid onset of action after an oral dose, can be given once daily, has a predictable anticoagulant response, and requires no monitoring. Given these attractive characteristics, 4 large scale trials have been completed that compare rivaroxaban to an existing strategy for VTE prophylaxis (enoxaparin) after major joint replacement surgery, the RECORD trials ${ }^{9-12}$. The trials differed by enoxaparin dose and the duration of enoxaparin and rivaroxaban therapy.

As the study design for the four trials was similar, we present the data for the RECORD 1 trial as an example ${ }^{9}$. In this study, authors randomly assigned 4,541 patients undergoing total hip replacement to either enoxaparin $40 \mathrm{mg}$ subcutaneously once daily or rivaroxaban $10 \mathrm{mg}$ orally once daily beginning on the day of surgery and continued for 35 days after surgery. The first dose of rivaroxaban was administered 6-8 hours after wound closure whereas the first dose of enoxaparin was given 12 hours before surgery. Exclusion criteria included bilateral hip surgery, pregnancy, active bleeding or high risk of bleeding, significant liver or renal disease, or an indication for long term anticoagulant therapy. All patients underwent venography the day after the last study drug dose. The primary outcome was a composite of deep

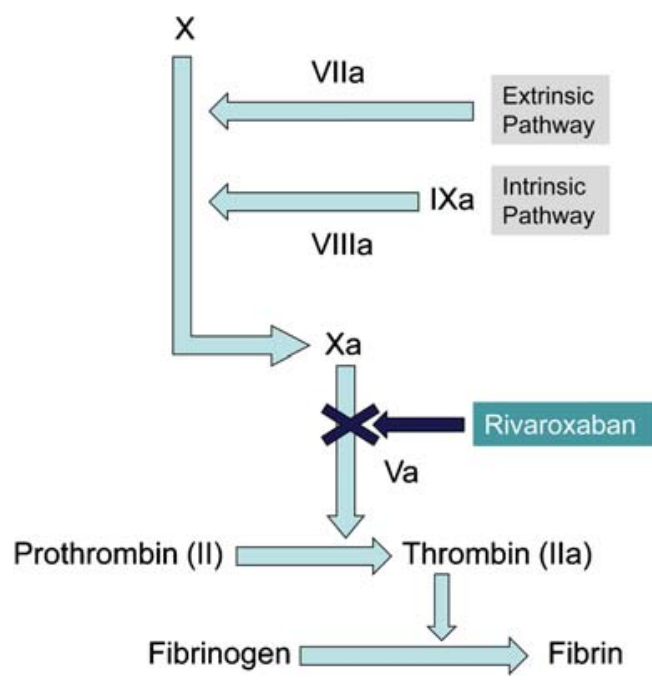

Figure 1. Site of action of rivaroxaban in the coagulation cascade. 
venous thrombosis, nonfatal pulmonary embolism, or death from any cause. A modified intention to treat analysis included all patients who had undergone the planned surgery, taken a study drug, and had received an adequate assessment for VTE. In this analysis, rivaroxaban was superior to enoxaparin; the primary outcome occurred in $1.1 \%$ and $3.7 \%$ of patients, respectively (relative risk reduction $70 \%$, CI $49 \%-82 \%$; $\mathrm{p}<$ 0.001 ). Major VTE rates were $0.2 \%$ vs. $2.0 \%$ (RRR $88 \%$, CI $66 \%-96 \%$; $<0.001)$. Rates of symptomatic VTE were substantially lower for both treatment groups and were not statistically different from each other $(0.3 \%$ vs. $0.5 \%$, respectively). Bleeding complications occurred in $6.0 \%$ of patients receiving rivaroxaban as compared to $5.9 \%$ for those receiving enoxaparin. Major bleeding was infrequent and comparable in both groups; rates were $0.3 \%$ versus $0.1 \%(p=0.18)$.

Efficacy outcomes across all four RECORD trials were similar. Table 1 summarizes the outcomes. Relative risk reductions associated with rivaroxaban use ranged from $31 \%-79 \%$. The number needed to treat to reduce one primary composite outcome (when compared to enoxaparin as the control) ranged from 11 to 39. Adverse events (not shown) were also comparable across the four RECORD trials with no clinically meaningful differences in any of the bleeding or nonbleeding complications between the two groups. Published rates of liver enzyme elevations (ALT and AST) during and after treatment were comparable between the two groups in all four trials. These efficacy and safety data are based on a large database of 12,729 patients.

Rivaroxaban represents an important step forward in VTE prophylaxis after major orthopedic surgery. It is more effective than low molecular weight heparin at reducing VTE events and has comparable rates of bleeding complications. It is an oral medication that requires no laboratory monitoring. It is this feature, along with the superior comparative efficacy, that represents a potential revolution in our management of anticoagulation after orthopedic surgery. Patient acceptance of this approach is likely to be high as no injections are required, and there will be no need for frequent blood draws and phone calls to anticoagulation clinics to adjust dose.

Rivaroxaban is safe in short term use; however no safety data exist for use beyond 35 days. It is possible that other adverse effects could emerge in longer term use; the drug will likely be initially approved only for short term use after orthopedic surgery. Rivaroxaban is currently in use in Europe and Canada and is under review by the U.S. FDA. In March 2009, an FDA advisory panel voted to recommend approval of the drug; however in May 2009 the FDA elected to delay approval and seek more information from the manufacturer including post marketing surveillance information from countries that have already approved the use of the drug.
The manufacturer intends to submit a full response to the FDA by the end of 2009 .

The evidence base for the use of rivaroxaban in settings other than surgical VTE prophylaxis is limited. Early phase II studies of its use in the treatment of deep venous thrombosis ${ }^{13}$ and acute coronary syndrome ${ }^{14}$ have been published; these reports support further study but must be considered preliminary. Studies of its use for stroke prevention in atrial fibrillation, treatment of pulmonary embolus, and VTE prophylaxis in medically ill patients are underway ${ }^{15}$. If further studies are supportive, rivaroxaban has the potential to replace warfarin for many indications. However, if rivaroxaban is approved for use in the U.S., clinicians must be extremely cautious and avoid the temptation to use it for off-label use pending further study as no long term safety data exist and patients with other indications for anticoagulant therapy may differ in important ways from those in the published orthopedic studies.

\section{Telcagepant}

Migraine affects $18 \%$ of women and $6 \%$ of men and is a source of substantial suffering and decreased productivity through lost school and work days. Even with effective pharmacologic and lifestyle preventive strategies, most migraineurs will have breakthrough headaches that require acute therapy. Response rates for medications used for acute treatment are typically $50-70 \%$; some patients are non responders to all available abortive agents. In addition, side effects may limit use of abortive therapies (e.g. dyspepsia from NSAIDs) and contraindications may prevent their safe use in other patients (e.g. uncontrolled hypertension precluding use of a triptan). Novel treatment strategies with similar or greater efficacy but fewer side effects would allow effective acute treatment for a larger proportion of migraine sufferers.

The exact pathophysiologic mechanisms of migraine are a subject of study and debate. Potential mechanisms include vascular dilatation, cutaneous allodynia, and neurogenic inflammation. This last mechanism has led to the study of several neuropeptides that are released from sensory neurons during the course of a migraine attack. These include substance $\mathrm{P}$, neurokinin $\mathrm{A}$, and calcitonin gene related peptide (CGRP). CGRP-containing sensory nerve fibers innervate vascular smooth muscle in the cerebral circulation and also other neurons. The observations that CGRP levels in the cerebral vascular circulation increase during a migraine attack, and that intravenous infusion of CGRP can trigger a migraine attack $^{16}$, prompted development of drugs that antagonize the effect of CGRP. As CGRP antagonism does not cause vasoconstriction, there is the potential for a drug that works through

Table 1. Primary Results from the RECORD Trials ${ }^{a}$

\begin{tabular}{|c|c|c|c|c|c|c|}
\hline & \multirow[t]{2}{*}{ Surgery } & \multirow[t]{2}{*}{$\mathrm{N}$} & \multicolumn{2}{|c|}{ Composite Events \% } & \multirow[t]{2}{*}{ RRR $(95 \% \mathrm{Cl})$} & \multirow[t]{2}{*}{ NNT $(95 \% \mathrm{Cl})$} \\
\hline & & & Rivaroxaban & Enoxaparin & & \\
\hline RECORD 1 & THA & 4541 & 3.7 & 1.1 & $70 \%$ (49\%-82\%) & 39 (27-67) \\
\hline RECORD 2 & THA & 2509 & 9.3 & 2.0 & $79 \%$ (65-87\% & $14(11-19)$ \\
\hline RECORD 3 & TKA & 2531 & 18.9 & 9.6 & $49 \%(34-60 \%)$ & $11(8-17)$ \\
\hline RECORD 4 & TKA & 3148 & 10.1 & 6.9 & $31 \%$ (8-49\%) & $38(18-143)$ \\
\hline
\end{tabular}

${ }^{a_{T H}}$ - total hip arthroplasty, TKA - total knee arthroplasty, RRR - relative risk reduction, CI - confidence interval, NNT - number needed to treat. Adapted with permission from Eikelboom JW and Weitz JI. Lancet 2008;372:6-8 
this mechanism to have no cardiovascular side effects. The first CGRP antagonist, olcegepant, proved to be effective as an acute treatment, but required intravenous infusion ${ }^{17}$.

Telcagepant is an oral CGRP receptor antagonist. Two early phase trials have evaluated its role in the acute treatment of migraine ${ }^{18,19}$. In the first dose finding phase II trial, authors randomly assigned 420 patients with migraine with or without aura to placebo, rizatriptan $10 \mathrm{mg}$, or one of seven doses of telcagepant (ranging from $25 \mathrm{mg}$ to $600 \mathrm{mg}$ ) followed by a second optional dose which was placebo for those originally receiving an active drug, and an active drug for those whose first dose was placebo. The primary outcome measure was pain relief at 2 hours. The four lowest dose arms were later discontinued due to lack of efficacy. Response rates were comparable for the three higher doses of telcagepant (300 mg, $400 \mathrm{mg}, 600 \mathrm{mg}$ ) and rizatriptan $10 \mathrm{mg}$. Two-hour pain relief was $68 \%, 48 \%, 68 \%$, and $70 \%$, respectively. There was no dose response relationship. Response to placebo was $46 \%$ illustrating the importance of using placebo arms in treatment trials for migraine.

This led to a phase III trial of similar design of 1,380 patients with migraine who were in good general health and who were free of cardiovascular disease or uncontrolled hypertension ${ }^{19}$. Eighty-five percent were women, $15 \%$ were taking migraine prophylaxis, and $45 \%$ usually used a triptan as an acute treatment. The random assignment was to telcagepant $150 \mathrm{mg}$ or $300 \mathrm{mg}$, zolmitriptan $5 \mathrm{mg}$, or placebo. This study had 5 coprimary endpoints measured at 2 hours after treatment: pain freedom or pain relief, and absence of phonophobia, photophobia, or nausea. Pain relief at 2 hours for telcagepant $150 \mathrm{mg}$, telcagepant $300 \mathrm{mg}$, zolmitriptan $5 \mathrm{mg}$, and placebo was $49.8 \%$ vs. $55.0 \%$ vs. $56.4 \%$ vs. $27.7 \%$, respectively. Pain freedom at 2 hours for the four groups was $17.2 \%$ vs. $26.9 \%$ vs. $31.3 \%, 9.6 \%$, respectively. Figure 2 displays response rates through 24 hours after dosing which was a secondary endpoint. Importantly, the side effect profile for telcagepant was more favorable than that of the currently available treatment, zolmitriptan. The rate of any adverse effect within 48 hours was similar for both doses of telcagepant and placebo ( $28 \%$ vs. $34 \%$ vs. $31 \%$ ), respectively, and was lower than that for zolmitriptan (50\%). While no statistical analysis was performed, side effects that were substantially less common for telcagepant than for zolmitriptan included dizziness, dry mouth, fatigue, paresthesia, throat tightness, and chest discomfort.

In preliminary studies, telcagepant appears to be as effective as a triptan in the acute treatment of migraine. It has fewer side effects including those potentially related to vasoconstriction. Its mechanism of action and clinical results to date suggest that it may be safe for patients with cardiovascular disease or risk factors, however such patients were excluded from the clinical trials so further study will be necessary to determine if this proves to be true. In April 2009, a small study of patients taking daily telcagepant (rather than episodically) was stopped due to reports of increased liver enzymes. In published trials, this did not occur among patients taking telcagepant for intermittent use. As of September 2009, the drug has not yet been submitted to the FDA. Pending further study of the potential for liver side effects, telcagepant has the potential to become a useful addition to currently available acute treatments for migraine.

\section{PHARMACOLOGIC TREATMENT UPDATES FOR PRIMARY CARE}

Every year, primary care clinicians must assimilate current recommendations about the most effective use of existing drugs. This year, we discuss recent literature reviews on the management of hyperglycemia in type 2 diabetes, care of the breast cancer survivor, and the treatment of hypertension.

\section{Management of Hyperglycemia in Type 2 Diabetes}

Aggressive lowering of glucose in patients with newly diagnosed type 2 diabetes reduces microvascular complications.

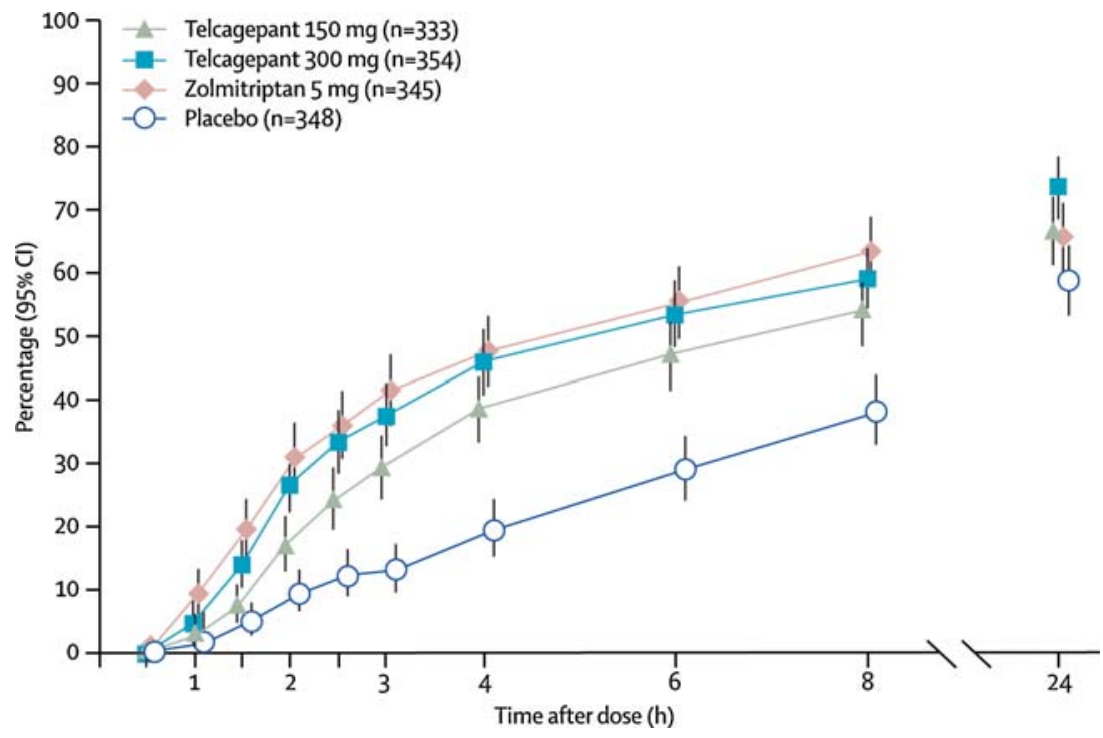

Figure 2. Pain freedom rate for up to 24 hours after acute migraine dosing. Reprinted with permission from Ho TH et al. Lancet 2008;372:2115-2123. 
Since type 2 diabetes is a progressive illness, patients will commonly require additional glucose lowering medications over time. The American Diabetes Association (ADA) and the European Association for the Study of Diabetes published a useful consensus algorithm in 2009 for the initiation and adjustment of therapy for hyperglycemia in type 2 diabetes ${ }^{20}$. The authors recommend a glycemic goal of Alc $<7 \%$ based on trials such as the UK Prospective Diabetes Study (UKPDS) ${ }^{21,22}$ that showed a reduction in microvascular and neuropathic complications with reduction in Alc $<7 \%$. Clinical judgment must prevail; the goal of an A1c $<7 \%$ may not be appropriate for some patients. The clinician must consider factors like the patient's life expectancy, and risk from hypoglycemia before deciding to intensify treatment. There are potential risks to aggressive treatment. In the recent ACCORD study ${ }^{23}$, which aimed to achieve an Alc of $<6 \%$, patients randomly assigned to tight control had higher cardiovascular mortality rates than those assigned to usual care (Alc goal 7.0-7.9\%). In the ADVANCE trial, which aimed to achieve an Alc of $<6.5 \%$, there was no excess mortality among patients randomly assigned to tight control, but no CVD benefit either, and more frequent severe hypoglycemia ${ }^{24}$.

The consensus algorithm consists of three management steps beginning with initiation of therapy at the time of diagnosis of type 2 diabetes. An underlying premise of the algorithm is that the patient will commit to self-monitoring of blood glucose to guide adjustments in treatment.

Step one occurs at the time of diagnosis and has two components: 1) lifestyle intervention including assessment by a dietician, and 2) starting metformin at the time of diagnosis. The decision to start metformin at the time of diagnosis reflects the ADA's acknowledgement that lifestyle interventions are insufficient for most patients and that early aggressive treatment is beneficial. This represents a shift from the customary paradigm and will substantially change clinical practice.

Currently, most guidelines use serum creatinine level to determine eligibility for metformin. The 2009 PDR states that patients should not receive metformin if the creatinine is $\geq 1.5 \mathrm{mg} / \mathrm{dl}$ in a man or $\geq 1.4 \mathrm{mg} / \mathrm{dl}$ in a woman. This is due to higher rates of the rare complication of lactic acidosis in patients with renal impairment. The new ADA guideline recommends use of the estimated glomerular filtration rate (eGFR) calculated from the Modification of Diet in Renal Disease (MDRD) formula, instead of the serum creatinine ${ }^{25}$. The researchers propose that an $\mathrm{eGFR}<30 \mathrm{ml} /$ minute is an absolute contraindication to metformin treatment, while an eGFR 30-59 ml/minute should alert clinicians to consider other risk factors for lactic acidosis such as hypoxia or hepatic impairment before initiating or continuing treatment. The use of the eGFR will enable more males to remain eligible for metformin treatment, and will more accurately assess renal impairment in the elderly.

The ADA recommends starting metformin $500 \mathrm{mg}$ once or twice daily, or $850 \mathrm{mg}$ once daily. One can then increase the daily dose by $500 \mathrm{mg}$ once a week or $850 \mathrm{mg}$ every other week. Recommended maximum doses are $2000 \mathrm{mg}$ per day, divided into two daily doses, or 2500-2550 mg per day, divided into three daily doses. The advantages of metformin include lowering of A1C by up to $1.5 \%$, no weight gain, no hypoglycemia, high acceptance by patients, and low cost. The main disadvantage is the occurrence of dose-limiting gastrointestinal side effects. More than $10 \%$ of patients taking metformin develop gastrointestinal side effects such as diarrhea. Switching from the immediate release metformin to an extended release preparation often decreases this side effect. If this preparation change does not work, it is necessary to decrease the dose of metformin to a level that is tolerated by the patient.

The ADA algorithm calls for checking the Alc after three months of treatment with lifestyle modification and metformin, and moving on to step 2 if the Alc is $\geq 7 \%$. Step 2 is to add a second medication, either insulin or a sulfonylurea. Other therapies such as pioglitazone or exenatide are less well validated. Pioglitazone has the advantage of not causing hypoglycemia and the disadvantages of increased risk for edema, congestive heart failure, weight gain, and bone loss. The ADA does not recommend rosiglitazone because it may increase cardiovascular risk $^{26}$. Exenatide does not cause hypoglycemia but is expensive, requires a twice daily injection, and may cause nausea and vomiting in more than $10 \%$ of patients. This is thought to be the main reason for treatment associated weight loss. The ADA does not include the DPP-4 inhibitors as an option for Step 2 because their long-term safety is not established, and they are expensive.

The ADA recommends insulin as the second medication if the Alc is $>8.5 \%$ or if symptomatic hyperglycemia is present. The recommended approach is bedtime intermediate or long-acting insulin at a dose of 10 units, or 0.2 units per $\mathrm{kg}$ with emphasis on the importance of teaching efficient selftitration of insulin dose. Table 2 summarizes the ADA approach to self-titration of insulin. The advantage of insulin is that the dose can be increased as much as is needed to achieve glycemic control. Disadvantages are the need for injection, and the potential for hypoglycemia and weight gain. Sulfonylureas have both pros and cons as a treatment modality. On the positive side, they lower the Alc by about 1.5\%. Significant disadvantages of sulfonylureas are weight gain and the potential for hypoglycemia. This occurs more often in the elderly and is more common with glyburide than with glipizide. Glycemic benefits are nearly fully realized at half-maximal doses of the sulfonylureas, e.g. glipizide $10 \mathrm{mg}$ per day. In general, clinicians should avoid the use of higher doses.

If the Alc remains $\geq 7 \%$ after 3 months on step 2 treatment, the clinician should move to the next step. Step 3 is to start or intensify insulin treatment, and to stop sulfonylurea treatment, as it is not synergistic with insulin and increases the risk for hypoglycemia. The ADA does not recommend adding a third oral agent to metformin and sulfonylurea as no data suggest that a 3 drug oral regimen is as effective as insulin,

Table 2. American Diabetes Association (ADA) Approach to Self-titration of Insulin

\begin{tabular}{l}
\hline \hline Approach \\
\hline 1. $\quad \begin{array}{c}\text { Measure the fasting glucose daily, with a goal of } 70-130 \text { for the } \\
\text { fasting glucose. }\end{array}$ \\
2. Increase insulin dose by 2 units every 3 days until the fasting \\
levels are at goal. \\
3. Increase the insulin by 4 units every 3 days if the fasting glucose is \\
$>180$.
\end{tabular}

Adapted with permission from Nathan DM, Buse JB, Davidson MB, et al. Diabetes Care 2009;32:193-203 
and it is much more expensive than treatment with insulin and metformin.

To intensify insulin treatment, the patient should first titrate the bedtime insulin dose until the fasting glucose is at the target of 70-130. The next step is to determine the optimal time for a second injection. If the serum glucose is highest before lunch, add an injection of rapid acting insulin at breakfast. If the pre-dinner glucose is the highest value, either add NPH insulin at breakfast or a rapid-acting insulin at lunch. If the pre-bedtime glucose is the most elevated, add rapid-acting insulin at dinner. Start with a dose of 4 units and teach the patient to adjust the dose by 2 units every 3 days until the glucose is at the target level of 70-130. Check the Alc again after 3 months. If it remains $\geq 7$, recheck the pre-lunch, dinner and bedtime glucoses. If one of these values remains $>130$, add a 3rd injection. If the Alc remains $\geq 7$ after an additional 3 months, check the 2 hour postprandial glucoses with a goal postprandial glucose of $<180$. Use this information to adjust the preprandial insulin dose.

This new algorithm emphasizes the importance of ongoing timely, aggressive management of hyperglycemia in type 2 diabetic patients. It provides a clear, simple, stepwise approach to management that can overcome therapeutic inertia by both clinician and patient and can lead to optimal glycemic control.

\section{Treatment Updates: Care of the Breast Cancer Survivor}

Breast cancer is the commonest cancer in women in U.S. There are currently two and a half million breast cancer survivors in the U.S. ${ }^{27}$. Primary care clinicians should be prepared to evaluate and treat the major problems that interfere with quality of life: menopausal symptoms, sexual dysfunction, and bone loss.

Menopausal symptoms occur frequently in the breast cancer patient. Causes include the following: 1) occurrence of natural menopause, 2) cessation of post-menopausal hormone treatment, 3) effects of endocrine therapy for estrogen receptor and progestin receptor positive breast cancer, 4) premature ovarian failure caused by chemotherapy, and 5) oophorectomy as part of breast cancer treatment. A recent practical clinical review addresses the assessment and management of these symptoms $^{28}$. Up to $20 \%$ of breast cancer patients consider stopping or actually stop endocrine treatment for their cancer because of hot flushes ${ }^{29}$. Nonpharmacologic treatments can ameliorate hot flushes. Studies of acupuncture show promising results. One RCT showed that acupuncture was more effective than placebo though not as efficacious as estrogen ${ }^{30}$. Another trial showed that acupuncture may improve sleep quality $^{31}$. Paced respirations and relaxation techniques are helpful, but require training ${ }^{32}$. A recent study of hypnosis showed a $59 \%$ decrease in hot flushes ${ }^{33}$.

Pharmacologic therapies include treatment with SSRIs and SNRIs. Table 3 summarizes the efficacy of pharmacologic treatment of hot flushes. Subjects had a baseline frequency of at least two bothersome hot flushes per day, with a mean of seven per day. Controlled release paroxetine is the most effective SSRI. In one report, the $12.5 \mathrm{mg}$ and $25 \mathrm{mg}$ controlled release doses were similarly effective in reducing hot flashes when compared to placebo. There were fewer side effects with the lower dose: $20 \%$ vs. $31 \%{ }^{34}$. Fluoxetine is effective ${ }^{34}$ while studies of citalopram, sertraline and mirtazapine have shown
Table 3. Treatment of Hot Flushes

\begin{tabular}{lllll}
\hline \hline \multirow{2}{*}{ Drug } & Daily dose & \multicolumn{3}{l}{ \% Reduction in hot flushes } \\
\cline { 3 - 5 } & $\begin{array}{l}\text { Active } \\
\text { treatment }\end{array}$ & Placebo & P value \\
\hline Paroxetine $\mathrm{CR}^{34}$ & $12.5 \mathrm{mg}$ & 62 & 38 & 0.007 \\
& $25 \mathrm{mg}$ & 65 & 38 & 0.03 \\
Fluoxetine $^{35}$ & $20 \mathrm{mg}$ & 50 & 36 & 0.02 \\
Venlafaxine $^{36}$ & $37.5 \mathrm{mg}$ & 37 & 27 & 0.008 \\
& $75 \mathrm{mg}$ & 61 & 27 & $<0.0001$ \\
Gabapentin $^{37}$ & $900 \mathrm{mg}$ & 49 & 21 & 0.0001 \\
Clonidine $^{38}$ & $0.1 \mathrm{mg}$ & 38 & 24 & 0.006 \\
\hline
\end{tabular}

${ }^{a}$ From a baseline of at least two (mean=7) bothersome hot flushes per day

mixed results ${ }^{39-41}$. The SNRIs can also be helpful. Venlafaxine has the best efficacy data. Recent data demonstrate a possible interaction of the SSRIs and SNRIs with tamoxifen, interfering with the breakdown of tamoxifen to its active metabolite by inhibiting the enzyme CYP2D6 ${ }^{42}$. In vitro, paroxetine and fluoxetine are potent inhibitors of CYP2D $6^{43,44}$. However, there are no clinical data to date. The current recommendation is to avoid strong inhibitors of CYP2D6 for patients who are taking tamoxifen. Citalopram and venlafaxine may be the safest choices in patients who are taking tamoxifen ${ }^{45}$.

Gabapentin can also ameliorate hot flushes ${ }^{37}$. Advantages include no significant drug interactions, associated sexual dysfunction, or withdrawal symptoms. Disadvantages include dizziness, unsteadiness, and drowsiness in up to $20 \%$ of patients ${ }^{46}$. Clonidine can be helpful in patients with mildmoderate hot flushes. Effective strategies include $0.1 \mathrm{mg}$ orally once a day ${ }^{38}$ and $0.1 \mathrm{mg}$ transdermal patch once weekly ${ }^{47}$. Side effects include dry mouth, insomnia and drowsiness.

In summary, the most effective pharmacologic treatments for hot flushes are venlafaxine and paroxetine CR. Paroxetine CR should not be used in patients who are taking tamoxifen. If one of these drugs is ineffective, it is reasonable to try the other one. Gabapentin is an alternative treatment. There is no benefit to adding gabapentin to an SSRI or SNRI ${ }^{28}$. Clonidine is a reasonable option for the patient with mild symptoms.

Atrophic vaginitis is another common symptom in women with breast cancer. The increasing use of aromatase inhibitors, which cause more vaginal dryness than tamoxifen, will probably lead to an increase in the number of such patients. Treatment begins with nonhormonal agents: vaginal moisturizers used daily, and lubricants used during intercourse. These products help some women but are not as efficacious as topical estrogens $^{28}$.

Vaginal estrogen preparations include estradiol (available as vaginal ring, cream and tablets), conjugated estrogen (available as a cream) and estriol, a less potent estrogen which can be compounded into a cream by pharmacies but is not FDA approved. Recent studies have shown increased blood estradiol levels in aromatase inhibitor users within 2 weeks of starting estradiol vaginal tablets. There is concern that these increased estradiol levels may reduce the efficacy of the aromatase inhibitors, and therefore, should be avoided ${ }^{48}$. In contrast, use of the vaginal estradiol ring does not appear to increase blood estradiol levels ${ }^{49}$. Current recommendations are to consider, in collaboration with the treating oncologist, estradiol as low dose cream or vaginal ring, and to avoid use of the estradiol tablets $^{28}$ 
Sexual dysfunction is common in breast cancer patients. Causes include the impact of the diagnosis and treatment of breast cancer, and the use of SSRIs and SNRIs, which can reduce libido and cause anorgasmia ${ }^{28}$. Clinicians should ask direct questions, as patients may be reluctant to bring up this problem. For example, "Are you having intercourse?" and "are you satisfied with the frequency and quality of your sexual experience?" Treatment options include stopping the SSRI or SNRI, augmentation with bupropion ${ }^{50}$, and sex therapy ${ }^{28}$. Treatments that are not recommended include testosterone and the type 5 phosphodiesterase inhibitors such as sildenafil.

In addition to traditional risk factors, risk factors for bone loss among breast cancer survivors include age $\geq 65$; age $60-64$ with family history of osteoporosis, a non-traumatic fracture; postmenopausal state on an aromatase inhibitor; and premature menopause $^{51}$. In addition to adequate amounts of calcium and vitamin $\mathrm{D}$, and regular weight bearing exercise, high risk patients should receive annual bone densitometry testing. If the bone densitometry shows osteopenia, assess the patient's 10-year risk for fracture with the World Health Organization's fracture risk algorithm and calculator, the FRAX calculator, available at www. shef.ac.uk/FRAX. Consider treatment if the 10-year risk for hip fracture is $\geq 3 \%$, or the 10-year risk of a major osteoporotic fracture is $>20 \%{ }^{52,53}$. Women with existing osteoporosis should receive treatment according to current guidelines.

The bisphosphonates are the first line pharmacologic options for treatment of patients with osteoporosis or osteopenia and a high 10-year risk of fracture. Raloxifene is an option for the postmenopausal woman who is unable to tolerate a bisphosphonate. In addition to reducing the risk of spine fractures, it reduces the risk of invasive breast cancer. It does not protect against hip fractures ${ }^{54,55}$.

\section{Updates on Hypertension Treatment}

Two new recommendations for the treatment of hypertension treatment are to make chlorthalidone rather than hydrochlorothiazide our thiazide of choice, and to treat hypertension in healthy patients who are 80 years of age or older.

Current Treatment Guidelines from the Medical Letter recommend chlorthalidone based on data that it is more effective than hydrochlorothiazide (HCTZ) ${ }^{56}$. Chlorthalidone is twice as potent as HCTZ, has a longer half-life (30 hours vs. 8 hours), and is more effective in lowering night-time and 24 hour ambulatory blood pressure ${ }^{57}$.

The evidence for cardiovascular risk reduction is strongest for chlorthalidone. For example, chlorthalidone in doses of 12.5-25 mg daily reduced cardiovascular events in both the ALLHAT and SHEP trials ${ }^{58,59}$. In contrast, there is little evidence that HCTZ at the customary 12.5-25 mg daily dose confers a reduction in cardiovascular events. In clinical trials, benefit occurred only with higher doses of HCTZ or in combination with triamterene or amiloride ${ }^{60}$.

The recent ACCOMPLISH trial, a RCT of 11,506 high-risk patients with hypertension, was stopped early because of higher rates of adverse cardiovascular outcomes with benazepril plus HCTZ than for benazepril plus amlodipine ${ }^{61}$. This finding may have been due to higher overnight and/or 24 hour ambulatory pressures among patients who were randomly assigned to the HCTZ regimen. The results of the 24 hour ambulatory blood pressure monitoring from the ACCOMPLISH trial have not yet been published.
Hypokalemia occurred in $7 \%$ of patients in the ALLHAT trial $^{62}$. Hypokalemia may be more common with chlorthalidone than HCTZ due to its longer duration of action. A low salt diet decreases the risk of hypokalemia. Hypokalemia with either drug usually develops during the first two weeks of treatment ${ }^{63}$. In the absence of acute intercurrent illness, a stable patient with a normal serum potassium level after two weeks of treatment is not at risk for the late development of hypokalemia.

It is reasonable to consider initial monotherapy with chlorthalidone $12.5 \mathrm{mg}$ a day, rather than HCTZ. The clinician should repeat measurement of blood pressure and electrolytes after two weeks. At that time, it is safe to increase the dose of chlorthalidone to $25 \mathrm{mg}$ per day if needed.

As to the second new recommendation, the recent HYVET trial established the benefit of the treatment of hypertension in healthy patients who are 80 years of age or older ${ }^{64}$. In this RCT of 3845 healthy patients age 80 or older with systolic BP of $\geq 160$, patients received either indapamide, a long acting thiazide, and then perindopril if needed, to reach a target blood pressure of $\leq 150 / 80$, or placebo. The primary end point was stroke. At two years, the mean blood pressure while sitting was 15.0/6.1 $\mathrm{mm} \mathrm{Hg}$ lower in the active treatment group than in the placebo group. Treatment conferred a $30 \%$ reduction in the rate of stroke (95\% CI -1 to $51, \mathrm{p}=0.06$ ); the number needed to treat (NNT) to prevent stroke was 94 . There was a $21 \%$ reduction in death from any cause $(95 \%$ CI 4 to $35, \mathrm{p}=$ 0.02). The implication for practice is that treating high blood pressure is beneficial in healthy patients 80 years of age or older. It is possible that patients with multiple comorbidities might benefit less and have more adverse events; the HYVET trial excluded such patients.

Acknowledgments: We wish to thank Michelle Agresti, Pharm D for her review of the new drugs portion of the manuscript and helpful suggestions.

Conflicts of interest: Dr. Smetana serves as a consultant to SafeMed. Dr. Sillman reports no conflict of interest.

Corresponding Author: Gerald W. Smetana, MD; Division of General Medicine and Primary Care, Beth Israel Deaconess Medical Center, Harvard Medical School, Shapiro 621D, 330 Brookline Ave, Boston, MA, USA (e-mail: gsmetana@bidmc.harvard.edu).

\section{REFERENCES}

1. Drugs @ FDA. FDA approved drug products. (Accessed November 11, 2009, at http://www.accessdata.fda.gov/Scripts/cder/DrugsatFDA.

2. Rubins HB, Robins SJ, Collins D, et al. Gemfibrozil for the secondary prevention of coronary heart disease in men with low levels of high-density lipoprotein cholesterol. Veterans Affairs High-Density Lipoprotein Cholesterol Intervention Trial Study Group. N Engl J Med. 1999;341:410-8.

3. Boekholdt SM, Sacks FM, Jukema JW, et al. Cholesteryl ester transfer protein TaqIB variant, high-density lipoprotein cholesterol levels, cardiovascular risk, and efficacy of pravastatin treatment: individual patient meta-analysis of 13, 677 subjects. Circulation. 2005;111:278-87.

4. Thompson A, Di Angelantonio E, Sarwar N, et al. Association of cholesteryl ester transfer protein genotypes with CETP mass and activity, lipid levels, and coronary risk. JAMA. 2008;299:2777-88.

5. Barter PJ, Caulfield M, Eriksson M, et al. Effects of torcetrapib in patients at high risk for coronary events. N Engl J Med. 2007;357:2109-22.

6. Krishna R, Anderson MS, Bergman AJ, et al. Effect of the cholesteryl ester transfer protein inhibitor, anacetrapib, on lipoproteins in patients with dyslipidaemia and on 24-h ambulatory blood pressure in healthy 
individuals: two double-blind, randomised placebo-controlled phase I studies. Lancet. 2007;370:1907-14.

7. Bloomfield D, Carlson GL, Sapre A, et al. Efficacy and safety of the cholesteryl ester transfer protein inhibitor anacetrapib as monotherapy and coadministered with atorvastatin in dyslipidemic patients. Am Heart J. 2009;157:352-60 e2.

8. Geerts WH, Bergqvist D, Pineo GF, et al. Prevention of venous thromboembolism: American College of Chest Physicians Evidence-Based Clinical Practice Guidelines (8th Edition). Chest. 2008;133:381S-453S

9. Eriksson BI, Borris LC, Friedman RJ, et al. Rivaroxaban versus enoxaparin for thromboprophylaxis after hip arthroplasty. N Engl J Med. 2008;358:2765-75.

10. Kakkar AK, Brenner B, Dahl OE, et al. Extended duration rivaroxaban versus short-term enoxaparin for the prevention of venous thromboem bolism after total hip arthroplasty: a double-blind, randomised controlled trial. Lancet. 2008;372:31-9.

11. Lassen MR, Ageno W, Borris LC, et al. Rivaroxaban versus enoxaparin for thromboprophylaxis after total knee arthroplasty. N Engl J Med. 2008;358:2776-86.

12. Turpie AG, Lassen MR, Davidson BL, et al. Rivaroxaban versus enoxaparin for thromboprophylaxis after total knee arthroplasty (RECORD4): a randomised trial. Lancet. 2009;373:1673-80.

13. Buller HR, Lensing AW, Prins MH, et al. A dose-ranging study evaluating once-daily oral administration of the factor Xa inhibitor rivaroxaban in the treatment of patients with acute symptomatic deep vein thrombosis: the Einstein-DVT Dose-Ranging Study. Blood. 2008;112:2242-7.

14. Mega J, Braunwald E, Mohanavelu S, et al. Rivaroxaban versus placebo in patients with acute coronary syndromes (ATLAS ACS-TIMI 46): a randomised, double-blind, phase II trial. Lancet 2009.

15. U.S. National Institutes of Health. Clinical Trials.gov. Accessed June 25, 2009.

16. Lassen LH, Haderslev PA, Jacobsen VB, Iversen HK, Sperling B, Olesen J. CGRP may play a causative role in migraine. Cephalalgia. 2002;22:54-61.

17. Olesen J, Diener HC, Husstedt IW, et al. Calcitonin gene-related peptide receptor antagonist BIBN 4096 BS for the acute treatment of migraine. N Engl J Med. 2004;350:1104-10.

18. Ho TW, Mannix LK, Fan X, et al. Randomized controlled trial of an oral CGRP receptor antagonist, MK-0974, in acute treatment of migraine. Neurology. 2008;70:1304-12.

19. Ho TW, Ferrari MD, Dodick DW, et al. Efficacy and tolerability of MK0974 (telcagepant), a new oral antagonist of calcitonin gene-related peptide receptor, compared with zolmitriptan for acute migraine: a randomised, placebo-controlled, parallel-treatment trial. Lancet. 2008;372:2115-23

20. Nathan DM, Buse JB, Davidson MB, et al. Medical management of hyperglycemia in type 2 diabetes: a consensus algorithm for the initiation and adjustment of therapy: a consensus statement of the American Diabetes Association and the European Association for the Study of Diabetes. Diabetes Care. 2009;32:193-203.

21. Intensive blood-glucose control with sulphonylureas or insulin compared with conventional treatment and risk of complications in patients with type 2 diabetes (UKPDS 33). UK Prospective Diabetes Study (UKPDS) Group. Lancet 1998;352:837-53.

22. Effect of intensive blood-glucose control with metformin on complications in overweight patients with type 2 diabetes (UKPDS 34). UK Prospective Diabetes Study (UKPDS) Group. Lancet 1998;352:854-65.

23. Gerstein HC, Miller ME, Byington RP, et al. Effects of intensive glucose lowering in type 2 diabetes. N Engl J Med. 2008;358:2545-59.

24. Patel A, MacMahon S, Chalmers J, et al. Intensive blood glucose control and vascular outcomes in patients with type 2 diabetes. $\mathrm{N}$ Engl $\mathrm{J}$ Med. 2008;358:2560-72

25. Shaw JS, Wilmot RL, Kilpatrick ES. Establishing pragmatic estimated GFR thresholds to guide metformin prescribing. Diabet Med. 2007;24:1160-3.

26. Singh S, Loke YK, Furberg CD. Long-term risk of cardiovascular events with rosiglitazone: a meta-analysis. JAMA. 2007;298:1189-95.

27. What are the key statistics for breast cancer? American Cancer Society. 2009. (Accessed November 11, 2009, at http://www.cancer.org/docroot/ CRI/content/CRI_2_4_1X_What_are_the_key_statistics_for_breast_cancer 5.asp?sitearea=

28. Hickey M, Saunders C, Partridge A, Santoro N, Joffe H, Stearns V. Practical clinical guidelines for assessing and managing menopausal symptoms after breast cancer. Ann Oncol. 2008;19:1669-80.
29. Barron TI, Connolly R, Bennett K, Feely J, Kennedy MJ. Early discontinuation of tamoxifen: a lesson for oncologists. Cancer. 2007;109:832-9.

30. Wyon Y, Wijma $\mathbf{K}$, Nedstrand E, Hammar M. A comparison of acupuncture and oral estradiol treatment of vasomotor symptoms in postmenopausal women. Climacteric. 2004;7:153-64

31. Huang MI, Nir Y, Chen B, Schnyer $\mathbf{R}$, Manber R. A randomized controlled pilot study of acupuncture for postmenopausal hot flashes: effect on nocturnal hot flashes and sleep quality. Fertil Steril. 2006;86:700-10.

32. Irvin JH, Domar AD, Clark C, Zuttermeister PC, Friedman R. The effects of relaxation response training on menopausal symptoms. J Psychosom Obstet Gynaecol. 1996;17:202-7.

33. Elkins G, Marcus J, Stearns V, Hasan Rajab M. Pilot evaluation of hypnosis for the treatment of hot flashes in breast cancer survivors. Psychooncology. 2007; 16:487-92.

34. Stearns V, Beebe KL, Iyengar M, Dube E. Paroxetine controlled release in the treatment of menopausal hot flashes: a randomized controlled trial. JAMA. 2003;289:2827-34.

35. Loprinzi CL, Sloan JA, Perez EA, et al. Phase III evaluation of fluoxetine for treatment of hot flashes. J Clin Oncol. 2002;20:1578-83.

36. Loprinzi CL, Kugler JW, Sloan JA, et al. Venlafaxine in management of hot flashes in survivors of breast cancer: a randomised controlled trial. Lancet. 2000;356:2059-63.

37. Pandya KJ, Morrow GR, Roscoe JA, et al. Gabapentin for hot flashes in 420 women with breast cancer: a randomised double-blind placebocontrolled trial. Lancet. 2005;366:818-24.

38. Pandya KJ, Raubertas RF, Flynn PJ, et al. Oral clonidine in postmenopausal patients with breast cancer experiencing tamoxifen-induced hot flashes: a University of Rochester Cancer Center Community Clinica Oncology Program study. Ann Intern Med. 2000;132:788-93.

39. Suvanto-Luukkonen E, Koivunen R, Sundstrom H, et al. Citalopram and fluoxetine in the treatment of postmenopausal symptoms: a prospective, randomized, 9-month, placebo-controlled, double-blind study. Menopause. 2005; 12:18-26.

40. Gordon PR, Kerwin JP, Boesen KG, Senf J. Sertraline to treat hot flashes: a randomized controlled, double-blind, crossover trial in a general population. Menopause. 2006;13:568-75.

41. Biglia N, Kubatzki F, Sgandurra P, et al. Mirtazapine for the treatment of hot flushes in breast cancer survivors: a prospective pilot trial. Breast J. 2007;13:490-5.

42. Stearns V, Johnson MD, Rae JM, et al. Active tamoxifen metabolite plasma concentrations after coadministration of tamoxifen and the selective serotonin reuptake inhibitor paroxetine. J Natl Cancer Inst. 2003;95:1758-64

43. Preskorn SH, Shah R, Neff M, Golbeck AL, Choi J. The potential for clinically significant drug-drug interactions involving the CYP 2D6 system: effects with fluoxetine and paroxetine versus sertraline. J Psychiatr Pract. 2007;13:5-12.

44. Jin Y, Desta Z, Stearns V, et al. CYP2D6 genotype, antidepressant use, and tamoxifen metabolism during adjuvant breast cancer treatment. J Natl Cancer Inst. 2005;97:30-9.

45. Stearns V. Clinical update: new treatments for hot flushes. Lancet. 2007;369:2062-4.

46. Butt DA, Lock M, Lewis JE, Ross S, Moineddin R. Gabapentin for the treatment of menopausal hot flashes: a randomized controlled trial. Menopause. 2008;15:310-8.

47. Nelson HD, Vesco KK, Haney E, et al. Nonhormonal therapies for menopausal hot flashes: systematic review and meta-analysis. JAMA. 2006;295:2057-71.

48. Kendall A, Dowsett M, Folkerd E, Smith I. Caution: Vaginal estradiol appears to be contraindicated in postmenopausal women on adjuvant aromatase inhibitors. Ann Oncol. 2006;17:584-7.

49. Weisberg E, Ayton R, Darling G, et al. Endometrial and vaginal effects of low-dose estradiol delivered by vaginal ring or vaginal tablet. Climacteric. 2005;8:83-92.

50. Mathias C. Cardeal Mendes CM, Ponde de Sena E, et al. An open-label, fixed-dose study of bupropion effect on sexual function scores in women treated for breast cancer. Ann Oncol. 2006;17:1792-6.

51. Hillner BE, Ingle JN, Chlebowski RT, et al. American Society of Clinical Oncology 2003 update on the role of bisphosphonates and bone health issues in women with breast cancer. J Clin Oncol. 2003;21:4042-57.

52. Dawson-Hughes B, Tosteson AN, Melton LJ 3rd, et al. Implications of absolute fracture risk assessment for osteoporosis practice guidelines in the USA. Osteoporos Int. 2008;19:449-58. 
53. Tosteson AN, Melton LJ 3rd, Dawson-Hughes B, et al. Cost-effective osteoporosis treatment thresholds: the United States perspective. Osteoporos Int. 2008; 19:437-47.

54. Ettinger B, Black DM, Mitlak BH, et al. Reduction of vertebral fracture risk in postmenopausal women with osteoporosis treated with raloxifene: results from a 3-year randomized clinical trial. Multiple Outcomes of Raloxifene Evaluation (MORE) Investigators. JAMA 1999;282:637-45.

55. Cummings SR, Eckert S, Krueger KA, et al. The effect of raloxifene on risk of breast cancer in postmenopausal women: results from the MORE randomized trial. Multiple Outcomes of Raloxifene Evaluation. JAMA. 1999;281:2189-97.

56. Drugs for hypertension. Treatment Guidelines from the The Medical Letter 2009;7:1-10.

57. Ernst ME, Carter BL, Goerdt CJ, et al. Comparative antihypertensive effects of hydrochlorothiazide and chlorthalidone on ambulatory and office blood pressure. Hypertension. 2006;47:352-8.

58. ALLHAT Collaborative Research Group. Major cardiovascular events in hypertensive patients randomized to doxazosin vs. chlorthalidone: the antihypertensive and lipid-lowering treatment to prevent heart attack trial (ALLHAT). JAMA. 2000;283:1967-75.

59. Prevention of stroke by antihypertensive drug treatment in older persons with isolated systolic hypertension. Final results of the Systolic Hypertension in the Elderly Program (SHEP). SHEP Cooperative Research Group. JAMA 1991;265:3255-64.

60. Messerli FH, Bangalore S. Antihypertensive efficacy of aliskiren: is hydrochlorothiazide an appropriate benchmark? Circulation. 2009;119:371-3.

61. Jamerson K, Weber MA, Bakris GL, et al. Benazepril plus amlodipine or hydrochlorothiazide for hypertension in high-risk patients. N Engl J Med. 2008;359:2417-28.

62. Maronde RF, Milgrom M, Vlachakis ND, Chan L. Response of thiazideinduced hypokalemia to amiloride. JAMA. 1983;249:237-41.

63. Langford HG, Schlundt D, Levine K. Sodium restriction in hypertension. Compr Ther. 1984;10:6-11.

64. Beckett NS, Peters R, Fletcher AE, et al. Treatment of hypertension in patients 80 years of age or older. N Engl J Med. 2008;358:1887-98. 